\title{
Slow-light enhanced optomechanical interactions
}

\author{
Andrey A. Sukhorukov ${ }^{1}$, Yue Sun ${ }^{1,2, *}$, and Thomas P. White ${ }^{3}$ \\ ${ }^{1}$ Nonlinear Physics Centre, Centre for Ultrahigh-bandwidth Devices for Optical Systems, Research \\ School of Physics and Engineering, Australian National University, Canberra ACT 0200, Australia \\ ${ }^{2}$ Laser Physics Centre, Centre for Ultrahigh-bandwidth Devices for Optical Systems, Research \\ School of Physics and Engineering, Australian National University, Canberra ACT 0200, Australia \\ ${ }^{3}$ Centre for Sustainable Energy Systems, Research School of Engineering, \\ Australian National University, Canberra ACT 0200, Australia
}

\begin{abstract}
We discuss potential advantages of slow-light waveguides compared to cavity-based structures for enhancing opto-mechanical interactions. Then, we reveal that slow-light enhanced optical forces between side-coupled photonic-crystal nanowire waveguides can be flexibly controlled by introducing a relative longitudinal shift. We predict that close to the photonic band-edge, where the group velocity is reduced, the transverse force can be tuned from repulsive to attractive, and the force is suppressed for a particular shift value. Additionally the shift leads to symmetry breaking that can facilitate longitudinal forces acting on the waveguides, in contrast to unshifted structures where such forces vanish.
\end{abstract}

Keywords: Slow light, Optomechanics, Nanowire waveguide, Photonic crystal

\section{INTRODUCTION}

Development of reconfigurable photonic circuits utilizing gradient optical forces between micro- and nano-scale optical waveguides opens new possibilities for optical signal shaping and routing based on all-optical tuning of the structure geometry. ${ }^{1-3}$ Whereas the pump intensity determines the strength of the gradient force, the sign of the gradient force between two identical coupled waveguides or cavities is dependant on the modal symmetry, with even modes typically associated with attractive and odd modes with repulsive forces. ${ }^{4}$ This has been demonstrated in side-coupled waveguides, where the force can be tuned from attractive to repulsive by varying the phase of the incident light to excite modes of different symmetries. ${ }^{1,2}$

In this work, we overview our recent results on engineering slow-light waveguides to enhance opto-mechanical interactions. First, in Sec. 2 we discuss a potential advantages of using slow-light waveguides ${ }^{5,6}$ compared to cavities ${ }^{7}$ based on the optical bandwidth and scalability with the length of the waveguide. Then, in Sec. 3 we present analytical and numerical results demonstrating that slow-light enhanced optically induced forces between side-coupled photonic-crystal wire waveguides can be controlled by introducing a relative longitudinal shift, enabling the tuning of the transverse force from repulse to attractive, and force suppression for a particular shift value. The shift-induced symmetry breaking can also facilitate longitudinal forces, in contrast to unshifted structures where such forces vanish. Finally, we present conclusions in Sec. 4.

\section{SLOW-LIGHT VS. CAVITY ENHANCEMENT OF OPTOMECHANICAL EFFECTS}

Optomechanical interactions can be enhanced in photonic-crystal waveguides due to increased optical energy concentration in the slow-light regime ${ }^{5,6}$ or through light trapping in cavities. ${ }^{7}$ Here, we perform a simple estimate of how the optical force-induced static mechanical deformation scales with an increase of the waveguide length.

Further author information available at the Nonlinear Physics Centre web site: http://physics.anu.edu.au/nonlinear

Advances in Slow and Fast Light V, edited by Selim M. Shahriar, Frank A. Narducci,

Proc. of SPIE Vol. 8273, 827317 · (c) 2012 SPIE · CCC code: 0277-786X/12/\$18 · doi: 10.1117/12.914744

Proc. of SPIE Vol. $8273827317-1$ 


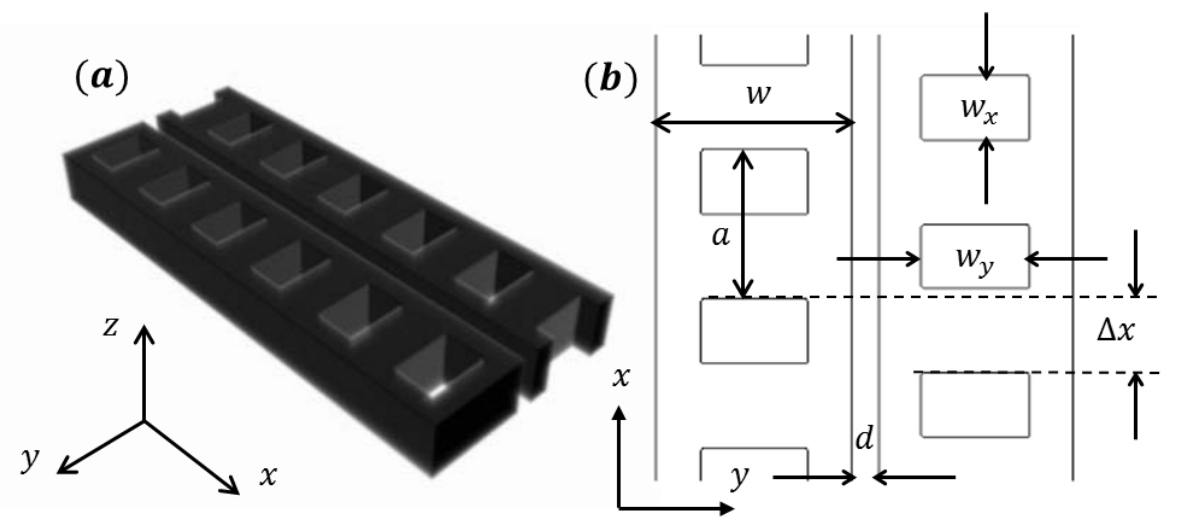

Figure 1. (a) 3D sketch of the longitudinally shifted side-coupled photonic-crystal waveguides. (b) top view of the structure.

We describe a suspended waveguide as an Euler-Bernoulli beam with supported ends. Then, the beam's deflection $y$ caused by the distributed force $f(x)$ in $y$ direction can be determined according to the following differential equation combined with the boundary conditions, ${ }^{8}$

$$
\begin{gathered}
\mathscr{E} \mathscr{I} \frac{d^{4} y}{d x^{4}}=f(x) \\
\left.\frac{d^{2} y}{d x^{2}}\right|_{x=0}=0,\left.\quad y\right|_{x=0}=0 \\
\left.\frac{d^{2} y}{d x^{2}}\right|_{x=L}=0,\left.\quad y\right|_{x=L}=0
\end{gathered}
$$

where $x$ is the centroid axis of the beam, $\mathscr{E}$ is the Young's modulus and $\mathscr{I}$ is the second moment of area with respect to $x$. We take the center of the left end of the beam as origin, then $L$ is the beam length. Here we neglect the nonlocal elasticity effects, ${ }^{9}$ as our aim here is to obtain simple and general approximate estimates.

For an optical cavity, the time-averaged force at a particular laser wavelength can be approximated as a Gaussian enveloped cosine shape,

$$
f_{c}(x)=\left\{C_{0} \cos [(x-L / 2) 2 \pi / a]+C_{2}\right\} \exp \left[-(x-L / 2)^{2} C_{1}^{-2} / 2\right] .
$$

where $C_{j}$ are parameters related to the cavity field, in particular $C_{1}$ is related to the cavity mode volume, and $a$ is a characteristic period of the photonic-crystal structure. For a slow-light waveguide, the force can be approximated as

$$
f_{s}(x)=S_{1} \cos [(x-L / 2) 2 \pi / a]+S_{0}
$$

where $S_{j}$ are parameters related to the slow-light mode field.

According to the equation of motion for Euler-Bernoulli beam, which can be approximately considered as uniformly loaded for slow-light waveguide and point loaded for a cavity structure, the deflection profile and the maximum deflection at the center are

$$
\begin{aligned}
\Delta y_{s} & =\frac{S_{0} x}{24 \mathscr{E} \mathscr{I}}\left(L^{3}-2 L x^{2}+x^{3}\right), \\
\Delta y_{c} & =\frac{C_{1} C_{2} \sqrt{2 \pi} x}{12 E I}\left(3 L^{2}-x^{2}\right), x \leq 0
\end{aligned}
$$

and the maximum deflections at the beam center are

$$
\begin{aligned}
\left(\Delta y_{s}\right)_{\text {max }} & =\frac{5 S_{0} L^{4}}{384 \mathscr{E} \mathscr{I}}, \\
\left(\Delta y_{c}\right)_{\max } & =\frac{C_{1} C_{2} \sqrt{2 \pi} L^{3}}{48 \mathscr{E} \mathscr{I}},
\end{aligned}
$$


where the subscripts $s$ and $c$ denote slow-light waveguide and cavity structures, respectively.

We now compare the maximum beam deflection in case of a cavity and slow-light waveguide, considering the same peak value of averaged force $S_{0}=C_{2}$, and the same length $L$ as well as the same stiffness $\mathscr{E} \mathscr{I}$ of the beam:

$$
\left(\Delta y_{s}\right)_{\max } /\left(\Delta y_{c}\right)_{\max }=\frac{5 S_{0} L^{4}}{384 \mathscr{E} \mathscr{I}} / \frac{C_{1} C_{2} \sqrt{2 \pi} L^{3}}{48 \mathscr{E} \mathscr{I}}=\frac{5 \cdot 48}{384 \sqrt{2 \pi}} \frac{L}{C_{1}}
$$

We see that as the beam is made longer, the beam deformation caused by the optical forces grows faster for slow-light waveguides compared to waveguides with a single cavity. On the other hand, slow-light waveguides can enable broadband operation, ${ }^{10,11}$ offering additional potential benefits compared to cavity-based structures. We also note that these estimates are performed for static deformations, and it is an interesting open problem how the mechanical vibrational modes scale with the length of slow-light waveguides.

\section{SLOW-LIGHT ENHANCED OPTICAL FORCES BETWEEN SHIFTED PHOTONIC-CRYSTAL NANOWIRE WAVEGUIDES}

We now analyze suspended wire waveguides containing a periodic array of rectangular holes as illustrated in Fig. 1(a), and investigate the effect of the longitudinal shift $\Delta x$. To provide a link with experimental platform we consider silicon nitride material (refractive index $n \simeq 2$ ) and choose the dimensions according to Ref. [7], but without a cavity since we aim to utilize slow-light enhancement of interactions which can be realized in defect-free photonic-crystal waveguides. ${ }^{5}$ The structure sketch is presented in Fig. 1. The period of the rectangular air hole array is $a$. The width and height of both waveguides are $w=7 a / 6$ and $h=2 a / 3$, respectively, and the space between them is $d=a / 6$. The width and length of the rectangular air holes are $w_{x}=0.445 a$ and $w_{y}=2 a / 3$ respectively.

\subsection{Effect of longitudinal shift on slow-light modes}

We use a plane-wave expansion method ${ }^{12}$ to calculate the supermode dispersion and the full 3D vectorial electromagnetic fields. In Figs. 2(a-c), we plot the dispersion curves for the first two transverse-magnetic (TM) bands close to the photonic band-edge, where the second band is the one we consider in the remainder of this paper. The corresponding group velocities for the second band are shown in Figs. 2(d-f). The plots are presented in dimensionless units: normalized frequency $\omega a / 2 \pi c$, normalized group velocity $v_{g} / c$, and normalized wavenumber $k a / 2 \pi$, where $\omega$ is optical angular frequency, $v_{g}$ is the group velocity, $k$ is the wavevector component along the waveguides, and $c$ is the speed of light in vacuum. The results are shown for three values of the longitudinal shift, as indicated in the figure caption. The group velocity vanishes at the photonic band-edge for all shift values, and this is a generally known feature. It is also accepted that light-matter interactions can be increased in the slow-light regime, including an enhancement of the optical gradient forces. ${ }^{5}$ However, as we demonstrate below, the introduction of a longitudinal shift has a nontrivial effect on slow-light enhanced optical forces, which can be attributed to breaking of symmetry of band-edge modes from odd (anti-symmetric) profiles in the absence of a shift [Figs. 2(g)], to complex phase profiles for shifted waveguides [Figs. 2(h,i)].

\subsection{General expressions for gradient forces}

We use the optical mode profiles to calculate the time-averaged gradient forces using the Maxwell stress tensor formulation $T_{\alpha \beta} \cdot{ }^{13}$ We take into account the structure periodicity and associated Bloch-mode symmetries to obtain the following simplified expressions for the distributed forces acting on the right waveguide in the transverse ( $y$-axis) and longitudinal $(x$-axis) directions,

$$
f_{y}=-\left.\int_{-\infty}^{+\infty} d z T_{y y}\right|_{y=0}, \quad f_{x}=-\left.\int_{-\infty}^{+\infty} d z T_{x y}\right|_{y=0} .
$$

Here, the coordinate axis origin $y=0$ lies in the middle between the nanowire waveguides, and

$$
\begin{aligned}
& \left.T_{y y}\right|_{y=0}=\left|E_{y}\right|^{2}-\left|E_{x}\right|^{2}-\left|E_{z}\right|^{2}+\left|H_{y}\right|^{2}-\left|H_{x}\right|^{2}-\left|H_{z}\right|^{2}, \\
& \left.T_{x y}\right|_{y=0}=E_{x}^{*} E_{y}+E_{x} E_{y}^{*}+H_{x}^{*} H_{y}+H_{x} H_{y}^{*} .
\end{aligned}
$$



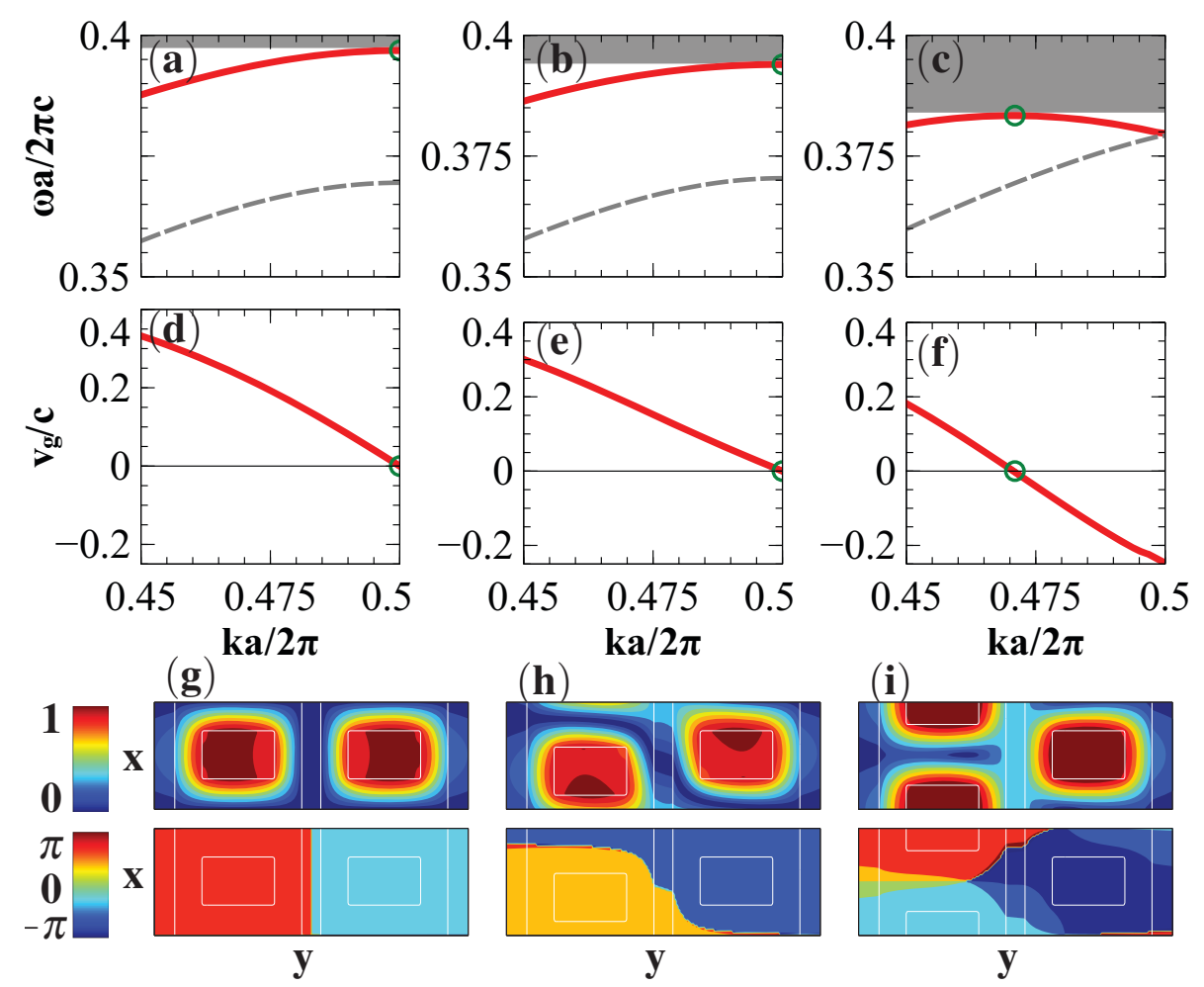

(h)
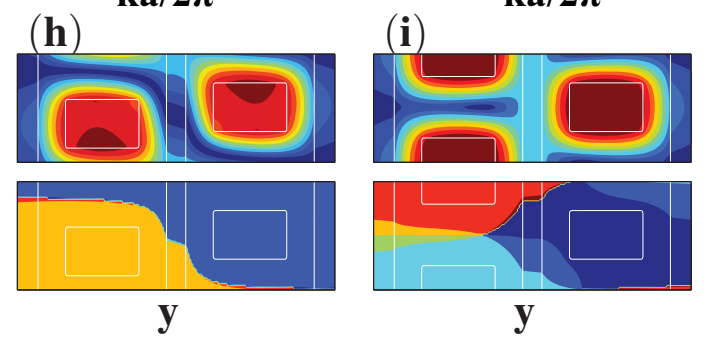

Figure 2. (Color online) TM mode properties in coupled waveguides with different longitudinal shifts: (a,d,g) $\Delta x=0$, (b,e,h) $\Delta x=0.15 a$, and (c,f,i) $\Delta x=0.5 a$. (a,b,c) Dispersion relations shown as normalized frequency vs. wavenumber for the first- (dashed) and secondlowest (solid line) TM bands near the band-edge; grey shading marks the band-gap. (d,e,f) Group velocities for the second-lowest band. (g,h,i) Mode profiles at the band-edge: intensity $|E|^{2}$ (top row) and phase (bottom row), white contours show an outline of the waveguides.

The force in the vertical direction vanishes, $f_{z}=0$, if the nanowires are free-standing or suspended high above a substrate. The gradient forces for the left waveguide have opposite values to those for the right waveguide. We also analyze the net forces over one period:

$$
F_{x, y}=\int_{-a / 2}^{a / 2} f_{x, y} d x
$$

For comparison between different structures, it is convenient to consider a normalized dimensionless force, ${ }^{4}$

$$
\widetilde{F}=F c / P,
$$

where $P$ is the power flow through the waveguides. In the absence of losses the optical energy stored within one period of the structure can be expressed as $U=P a /\left|v_{g}\right|$. As the group velocity is reduced close to the photonic band-edge, the value of $U$ increases, and the force value also grows as

$$
\widetilde{F} \sim\left|v_{g}\right|^{-1} .
$$

In order to compare forces in the slow-light regime for different structure parameters, we also consider a scaled force value

$$
\widetilde{F}\left|v_{g}\right| / c \equiv F\left|v_{g}\right| / P \text {. }
$$

\subsection{Transverse forces}

In the transverse direction, the optically induced force originates from the strong variation of the electromagnetic field due to the evanescent coupling between two waveguides. Most importantly, we show in Fig. 3(a) that the slow-light enhanced 

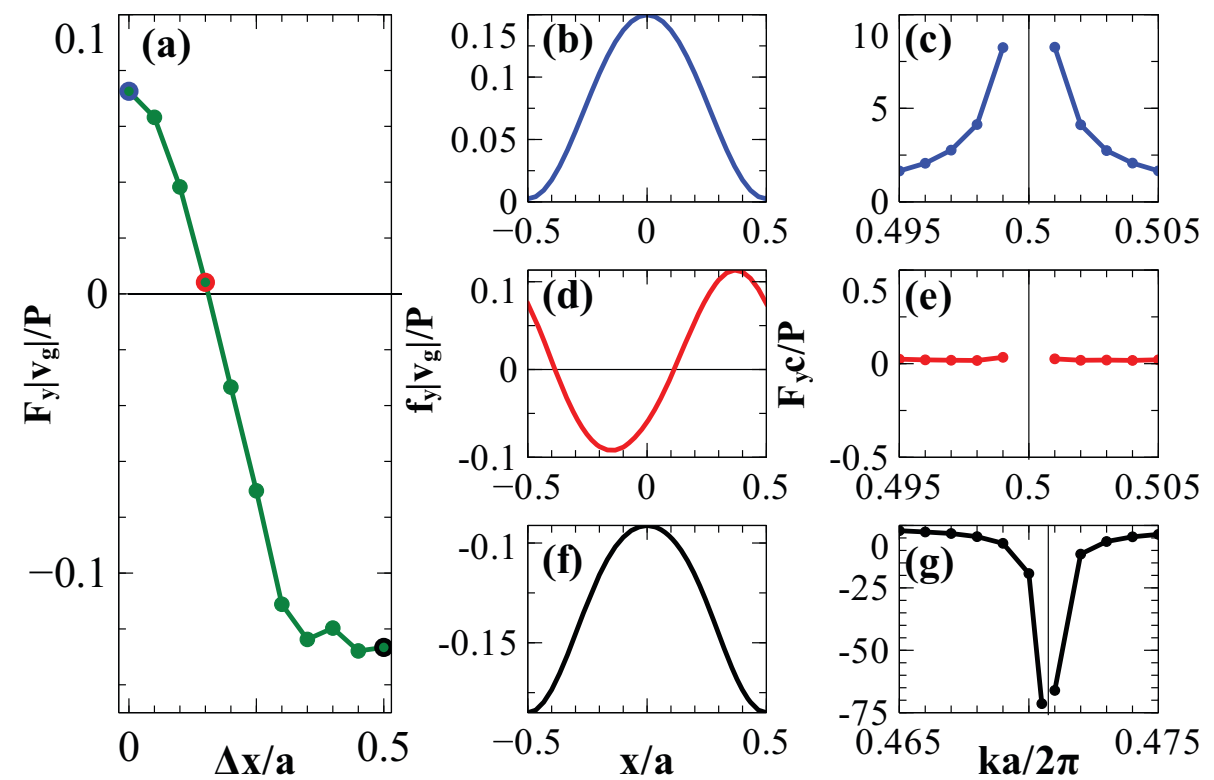

Figure 3. The force in the transverse $(+y)$ direction on the right waveguide. (a) The force per unit length per unit energy density vs. the longitudinal shift at the band-edge. (b-g) Forces for different longitudinal shifts: (b,c) $\Delta x=0$, (d,e) $\Delta x=0.15 a$ and (f,g) $\Delta x=0.5 a$. $(\mathrm{b}, \mathrm{d}, \mathrm{f})$ The distributed force along the waveguide per unit energy density at the band-edge. (c,e,g) Total force per unit length and unit power vs. the wavenumber near the photonic band-edge.

force at the photonic band edge changes from repulsive (positive value) to attractive (negative value) as the longitudinal shift is tuned from zero $(\Delta x=0)$ to half a period $(\Delta x=0.5 a)$.

In the absence of a longitudinal shift $(\Delta x=0)$, the band-edge mode has odd symmetry about the $y$-axis [Fig. 2(g)] which provides a repulsive force at all waveguide locations [Fig. 3(b)] in agreement with earlier studies for non-periodic waveguides ${ }^{4}$ and in periodic waveguides the force is additionally enhanced close to photonic band-edge, see Fig. 3(c). When a longitudinal shift is introduced, the left-right symmetry of the coupled waveguide structure is broken, and this leads to cross-coupling between odd and even supermodes. Consequently, the electromagnetic fields become a mixture of even and odd modes [Fig. 2(h,i)] and the distributed force along the $x$ direction changes its offset and peak-to-peak value, c.f. Figs. 3(b,d,f). As the longitudinal shift increases, the relative fraction of even supermodes gets larger, which explains the transition to transversal attraction between waveguides at larger shifts. In particular, the strongest slow-light enhanced attraction occurs for half a period shift $(\Delta x=0.5 a)$, when the distributed force is attractive at all waveguide locations, see Figs. 3(f,g). Quite interestingly, for an intermediate shift value of $\Delta x \simeq 0.15 a$, the transverse force is suppressed even in the slow-light regime, see Fig. 3(e), which happens because the distributed force oscillates between repulsive and attractive along the waveguide [Fig. 3(d)] providing zero average force along a structure period.

We estimate the characteristic values of the transverse optical forces, considering $100 \mathrm{~mW}$ optical power coupled into the waveguides and $1550 \mathrm{~nm}$ band-edge wavelength. Efficient coupling to slow-light modes can be achieved by introducing a taper structure. ${ }^{14}$ Then, the transverse force per unit length is $4.45 \mathrm{nN} / \mu \mathrm{m}$ for $\Delta x=0, a \simeq 617 \mathrm{~nm}, k a / 2 \pi=0.499$, and $v_{g} / c=0.0081$ [Fig. 3(c)]; $0.02 \mathrm{nN} / \mu \mathrm{m}$ for $\Delta x=0.15 a, a \simeq 612 \mathrm{~nm}, k a / 2 \pi=0.499$, and $v_{g} / c=0.0057$ [Fig. 3(e)]; and $-39.91 \mathrm{nN} / \mu \mathrm{m}$ for $\Delta x=0.5 a, a \simeq 596 \mathrm{~nm}, k a / 2 \pi=0.4705$, and $v_{g} / c=0.0018$ [Fig. 3(g)].

\subsection{Longitudinal forces}

In the longitudinal direction, the optical force also results from the strong electromagnetic field variation along the $y$ direction according to Eq. (7). The scaled force per unit length per unit energy density is shown in Fig. 4(a). We note that it has a maximum value at $\Delta x \simeq 0.3 a$ shift, while zero and half-period shifts correspond to zero force. In order to explain this force dependence, we use the general symmetry properties of electromagnetic fields in periodic photonic structures.

For zero or half-period shifts, the refractive index profile has a mirror symmetry with respect to a reflection transfor- 

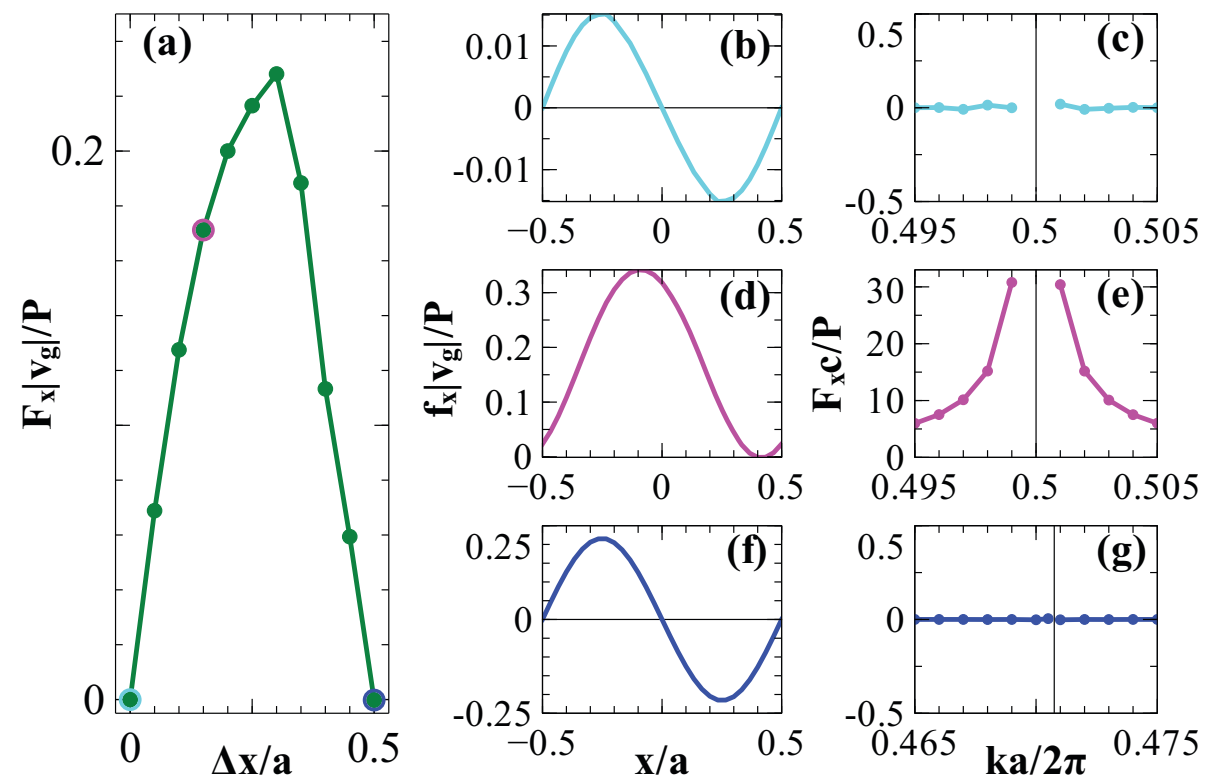

Figure 4. The force in the longitudinal $(+x)$ direction on the right waveguide. (a) The force per unit length per unit energy density vs. the longitudinal shift at the band-edge. (b-g) Forces for different longitudinal shifts: (b,c) $\Delta x=0,(\mathrm{~d}, \mathrm{e}) \Delta x=0.15 a$ and (f,g) $\Delta x=0.5 a$. $(\mathrm{b}, \mathrm{d}, \mathrm{f})$ The distributed force along the waveguide per unit energy density at the band-edge. (c,e,g) Total force per unit length and unit power vs. the wavenumber near the photonic band-edge.

mation $x \rightarrow-x$, and then it follows ${ }^{15}$ that Bloch wave profiles should satisfy relations

$$
\begin{aligned}
E_{y, z}(x, y, z ; k) & =E_{y, z}(-x, y, z ;-k) \exp \left(i \varphi_{1}\right), \\
H_{y, z}(x, y, z ; k) & =-H_{y, z}(-x, y, z ;-k) \exp \left(i \varphi_{1}\right), \\
E_{x}(x, y, z ; k) & =-E_{x}(-x, y, z ;-k) \exp \left(i \varphi_{1}\right), \\
H_{x}(x, y, z ; k) & =H_{x}(-x, y, z ;-k) \exp \left(i \varphi_{1}\right) .
\end{aligned}
$$

Additionally, for lossless structures with real refractive index profile, there is a symmetry related to time-reversal invariance: $^{15}$

$$
\begin{aligned}
& E_{x, y, z}^{*}(x, y, z ; k)=E_{x, y, z}(x, y, z ;-k) \exp \left(i \varphi_{2}\right), \\
& H_{x, y, z}^{*}(x, y, z ; k)=H_{x, y, z}(x, y, z ;-k) \exp \left(i \varphi_{2}\right) .
\end{aligned}
$$

In these expressions the phase parameters $\varphi_{1,2}$ can take 0 or $\pi$ values depending on the mode symmetry. Using these symmetry relations, the tensor component responsible for the longitudinal force according to Eqs. ( 7) can be transformed as:

$$
\begin{aligned}
T_{x y}=[ & -E_{x}(-x, y, z ; k) E_{y}(x, y, z ; k)+E_{x}(x, y, z ; k) E_{y}(-x, y, z ; k) \\
& \left.+H_{x}(-x, y, z ; k) H_{y}(x, y, z ; k)-H_{x}(x, y, z ; k) H_{y}(-x, y, z ; k)\right] \exp (i \varphi),
\end{aligned}
$$

where $\varphi=\varphi_{1}+\varphi_{2}$. It is then clear that

$$
T_{x y}(x, y, z ; k)=-T_{x y}(-x, y, z ; k),
$$

and accordingly the distributed longitudinal force is also an antisymmetric function of $x$,

$$
f_{x}(x ; k)=-f_{x}(-x ; k) .
$$

Indeed, the antisymmetric property of the distributed force is clearly visible in Figs. 4(b,f). Therefore, for structures with zero or half-period shifts the integral force over one period will always vanish, $F_{x} \equiv 0$, see the force dependence near the band-edge in Figs. 4(c,g).

For intermediate shift values, $0<\Delta x<0.5 a$, the structure's mirror symmetry is broken, resulting in a non-vanishing longitudinal force, see Figs. 4(d,e). For $100 \mathrm{~mW}$ optical power and $1550 \mathrm{~nm}$ band-edge wavelength, we estimate the force per unit length is $16.76 n N / \mu m$ for $\Delta x=0.15 a, a \simeq 612 \mathrm{~nm}, k a / 2 \pi=0.499$ and $v_{g} / c=0.0057$. 


\section{CONCLUSIONS}

In conclusion, the slow-light enhanced optical forces exhibit scalability vs. the waveguide length exceeding that associated with optical cavities. We also show that the forces between side-coupled photonic-crystal waveguides can be be controlled by a longitudinal shift. The shift enables force control beyond the possibilities of symmetric structures with unshifted waveguides. In the transverse direction, the longitudinal shift can be used to tune the optical gradient force from repulsive to attractive, and the force can be also suppressed. The shift gives rise to force in the longitudinal direction along the waveguide, whereas this force component is always absent in symmetric structures. We also note that in shifted structures, pairs of slow-light modes can co-exist at the photonic band-edge enabling slow-light tunneling between the two waveguides, ${ }^{16,17}$ which may offer additional possibilities for tailoring the forces. Overall, the longitudinal waveguide shifting presents new opportunities for controlling gradient forces which may contribute to the development of optomechanical circuits.

We acknowledge support from the Australian Research Council and NCI National Facility.

\section{REFERENCES}

1. M. Li, W. H. P. Pernice, and H. X. Tang, "Tunable bipolar optical interactions between guided lightwaves," Nature Photonics 3, 464-468 (2009).

2. J. Roels, I. De Vlaminck, L. Lagae, B. Maes, D. Van Thourhout, and R. Baets, "Tunable optical forces between nanophotonic waveguides," Nat. Nanotechnol. 4, 510-513 (2009).

3. M. Eichenfield, R. Camacho, J. Chan, K. J. Vahala, and O. Painter, "A picogram- and nanometre-scale photoniccrystal optomechanical cavity," Nature 459, 550-U79 (2009).

4. M. L. Povinelli, M. Loncar, M. Ibanescu, E. J. Smythe, S. G. Johnson, F. Capasso, and J. D. Joannopoulos, "Evanescent-wave bonding between optical waveguides," Opt. Lett. 30, 3042-3044 (2005).

5. J. Ma and M. L. Povinelli, "Effect of periodicity on optical forces between a one-dimensional periodic photonic crystal waveguide and an underlying substrate," Appl. Phys. Lett. 97, 151102-3 (2010).

6. A. Oskooi, P. A. Favuzzi, Y. Kawakami, and S. Noda, "Tailoring repulsive optical forces in nanophotonic waveguides," Opt. Lett. 36, 4638-4640 (2011).

7. J. Chan, M. Eichenfield, R. Camacho, and O. Painter, "Optical and mechanical design of a "zipper" photonic crystal optomechanical cavity,” Opt. Express 17, 3802-3817 (2009).

8. S. Timoshenko, Strength of materials, 3rd ed. (Van Nostrand, London, 1955).

9. T. Murmu and S. Adhikari, "Nonlocal transverse vibration of double-nanobeam-systems," J. Appl. Phys. 108, 083514-9 (2010).

10. T. F. Krauss, "Why do we need slow light?," Nature Photonics 2, 448-450 (2008).

11. S. A. Schulz, L. O'Faolain, D. M. Beggs, T. P. White, A. Melloni, and T. F. Krauss, "Dispersion engineered slow light in photonic crystals: a comparison,” J. Opt. 12, 104004-10 (2010).

12. S. G. Johnson and J. D. Joannopoulos, "Block-iterative frequency-domain methods for Maxwell's equations in a planewave basis," Opt. Express 8, 173-190 (2001).

13. J. D. Jackson, Classical Electrodynamics (Wiley, New York, 1998).

14. M. L. Povinelli, S. G. Johnson, and J. D. Joannopoulos, "Slow-light, band-edge waveguides for tunable time delays," Opt. Express 13, 7145-7159 (2005).

15. J. D. Joannopoulos, R. D. Meade, and J. N. Winn, Photonic Crystals: Molding the Flow of Light, 2nd ed. (Princeton University Press, Princeton, 2008).

16. S. W. Ha, A. A. Sukhorukov, K. B. Dossou, L. C. Botten, A. V. Lavrinenko, D. N. Chigrin, and Yu. S. Kivshar, "Dispersionless tunneling of slow light in antisymmetric photonic crystal couplers," Opt. Express 16, 1104-1114 (2008).

17. S. Ha, A. A. Sukhorukov, A. V. Lavrinenko, I. V. Shadrivov, D. A. Powell, and Yu. S. Kivshar, "Observation of tunneling of slow and fast electromagnetic modes in coupled periodic waveguides," Appl. Phys. Lett. 98, 061909-3 (2011). 